\title{
Functional outcome following quadriceps tendon lengthening in congenital dislocation of the knee, with special reference to extensor weakness
}

\author{
Alok Sud • Aashish Chaudhry • Anil Mehtani • \\ Akshay Tiwari $\cdot$ Deep Sharma
}

Received: 22 June 2009/Accepted: 7 November 2009/Published online: 24 November 2009

(C) Springer-Verlag 2009

\begin{abstract}
This study reports the incidence and proposes a probable cause of flexion deformity and extensor weakness following lengthening of quadriceps tendon for the congenital dislocation of the knee and also proposes a modification of the functional grading given in the literature to this effect. Seventeen knees in ten patients were treated with a follow-up from 3 to 8 years. Fifteen knees were grade III and were operated with quadriceps lengthening and anterior capsulotomy. Two knees were Grade II and were initially closed reduced, but operated later due to recurrence. The results were graded on a modification of functional grading system given in the literature [1]. There were excellent results in four, good in five and fair in eight knees. There was an extensor weakness in ten knees (average $12.2^{\circ}$ ) and flexion deformity $\left(15^{\circ}\right)$ in one. Eight patients could squat and all started walking independently by 10-20 months of age, except one. V-lengthening of the quadriceps tendon produced a satisfactory improvement in the knee function. The theoretical role of the gastrocnemius and hamstring muscles in abetting flexion deformity and extensor weakness is suggested.
\end{abstract}

Keywords Knee dislocation · Quadriceps

A. Sud $(\bowtie) \cdot$ A. Chaudhry · A. Mehtani · D. Sharma Department of Orthopedics, Lady Hardinge Medical College, U-V/116 C Shalimar Bagh, New Delhi 110088, India e-mail: avimukta2@gmail.com

A. Tiwari

Department of Orthopedics, Rajiv Gandhi Cancer Hospital, New Delhi, India

\section{Introduction}

Congenital dislocation of knee is a rare deformity $(0.017 /$ 1,000 live births) [2]. It is reported to occur usually in conjunction with multiple congenital anomalies like arthrogryposis multiplex congenita (AMC), Larsen syndrome, spina bifida, developmental dysplasia of hip (DDH), club foot, forefoot adduction deformity and calcaneovalgus foot deformities [3-5]. The underlying cause is a shortened and fibrotic quadriceps femoris muscle which does not allow any flexion at the knee joint rendering a highly unstable and useless knee joint [4, 6, 7]. Lengthening of the quadriceps tendon with anterior capsulotomy has been used successfully to produce a satisfactory result $[1,3,8]$. Most series do not elaborate upon the incidence of flexion deformity or extensor lag $[1,8,9]$. We report our results with $\mathrm{V}$-lengthening of quadriceps muscle tendon [3] in 15 Grade III and two Grade II knees with special reference to the functional outcome of the knee joint.

\section{Materials and methods}

Congenital dislocation of the knee was seen in ten patients (17 knees) over a period of 6 years (January 2000 through December 2006) at our Paediatric Orthopaedics Clinic. There were 15 knees with complete dislocation (Grade III) and two with subluxation (Grade II) [10]. Patients with Grade I knees $\left(-15^{\circ}\right.$ hyperextension to full flexion) were excluded from the study. Concomitant deformities were seen in eight patients.

All the patients, after informed consent, underwent an initial attempt of manipulation and closed reduction with serial plaster casts applied weekly. Initial reduction was 
achieved in two knees (Grade II) but failed in the rest. Recurrence of deformity was seen in both the knees treated conservatively within 6 months of the initial reduction. An open reduction of the knee joint following V-lengthening of the tendon of quadriceps muscle and anterior capsulotomy was carried out in all [3].

The limbs were held in above knee casts in $30^{\circ}$ flexion for 6 weeks after operation. Following this, the cast was removed and active range of motion was encouraged. Weight bearing was allowed at 8-12 weeks after operation when optimal strength and range of motion were achieved [3]. The operative and post-operative management changed according to specific needs of the child and the associated deformities. When multiple joints were involved, we mandated to correct the knee first as a good hip position could not be maintained with hyperextended knees. Similarly it was difficult to correct clubfoot deformities when knee could not be brought into flexion [9].

Those with clubfeet were treated, after lengthening of the quadriceps tendon, by a complete subtalar release at the same sitting. Those with DDH had closed reductions performed after achieving flexion at the knee by quadriceps lengthening.

A satisfactory outcome was defined as an active and useful flexion range with no flexion deformity, active extension at knee with no extensor weakness or deformity, pain free movement without instability and the ability to stand and walk without external support. We classified our results into excellent, good, fair and poor according to a previously reported grading system [1]. Excellent knee function had a full range of stable, painless motion; good, flexion to $90^{\circ}$ or more with slight instability or pain; fair, flexion of 45 to $90^{\circ}$ with mild instability or pain, and poor, flexion of less than $45^{\circ}$ with gross instability and pain. It was modified to include the presence of extensor lag, a flexion deformity or use of walking aids or braces. A deduction of one category was incurred if any one of these factors was present. Extensor weakness or lag was defined as an inability to achieve normal extension at knee joint actively. However, the knee could be extended passively. In flexion deformity, passive knee extension was also absent.

\section{Results}

There was an equal distribution of both sexes (five girls and five boys). There were seven patients with bilateral involvement. All patients presented between 2 and 25 months of age (average 6.1 months). Conservative management failed in all. The average age of operation was 10.4 months.

None had a family history of the deformity. However, two patients were siblings. The majority of infants were delivered normally through the vaginal route, with one breach delivery and two Caesarean sections. Eight infants had associated musculoskeletal deformities in one or more limbs-clubfoot (4 limbs), forefoot adduction (1 limb), postural calcaneovalgus deformity (3 limbs), congenital vertical talus (2 limbs), DDH (5 limbs), arthrogryposis multiplex congenital (4 children) and Down's syndrome in one child (Table 1).

When examined preoperatively, the range of motion at the affected knee in grade III cases was absent or minimal with tight anterior structures, restricting almost all flexion. In grade II knees, the range of motion after reduction was from hyperextension to about $45^{\circ}$ of flexion. The average range of motion obtained after quadriceps tendon repair at surgery was from hyperextension to $26^{\circ}$ of flexion. It subsequently increased in most cases to $90^{\circ}$ or greater (average $122^{\circ}$ ). All the patients except one (Case 10) were able to walk independently by 10-20 months of age (average 13.7 months, SD: 3.206) without external support. Case 10 started walking with braces at the age of 32 months. There was no anteroposterior instability in any patient. Although valgus was present in four patients, this was within physiological limits and no additional procedure was required.

At final follow-up, a flexion deformity of $15^{\circ}$ was present in one knee and a mean extensor lag of $12.2^{\circ}$ was present in eleven knees (in seven patients). However, no patient had problems in standing and walking.

There were four knees (in 3 patients) with excellent results, where the mean active range of motion was $-5^{\circ}$ to $140^{\circ}$. These children could squat freely and had painless stable knees with no flexion deformity or extensor lag. Five knees had good results where the mean active range of motion was from $+8^{\circ}$ to $136^{\circ}$, respectively. They could also squat and had no pain. Eight knees had fair results with a mean active range of motion from $+13.1^{\circ}$ to $105^{\circ}$. Mediolateral instability of less than $5 \mathrm{~mm}$ was present in only one knee (case 2) and this improved over time. None of the patients had any pain and were able to squat easily or with slight difficulty only. Despite having a range of motion of more than $90^{\circ}$, they were classified into good and fair categories due to the presence of a flexion deformity or extensor lag. However, all these knees could be passively extended with the exception of case 2 (left side) which had a flexion deformity of $15^{\circ}$.

\section{Discussion}

Conservative management by gradual stretching and serial casts is helpful in a younger child (usually less than 3 months) with an isolated dislocation; otherwise operative intervention may be required [1]. A relationship between age and the presence of multiple concomitant anomalies to the 


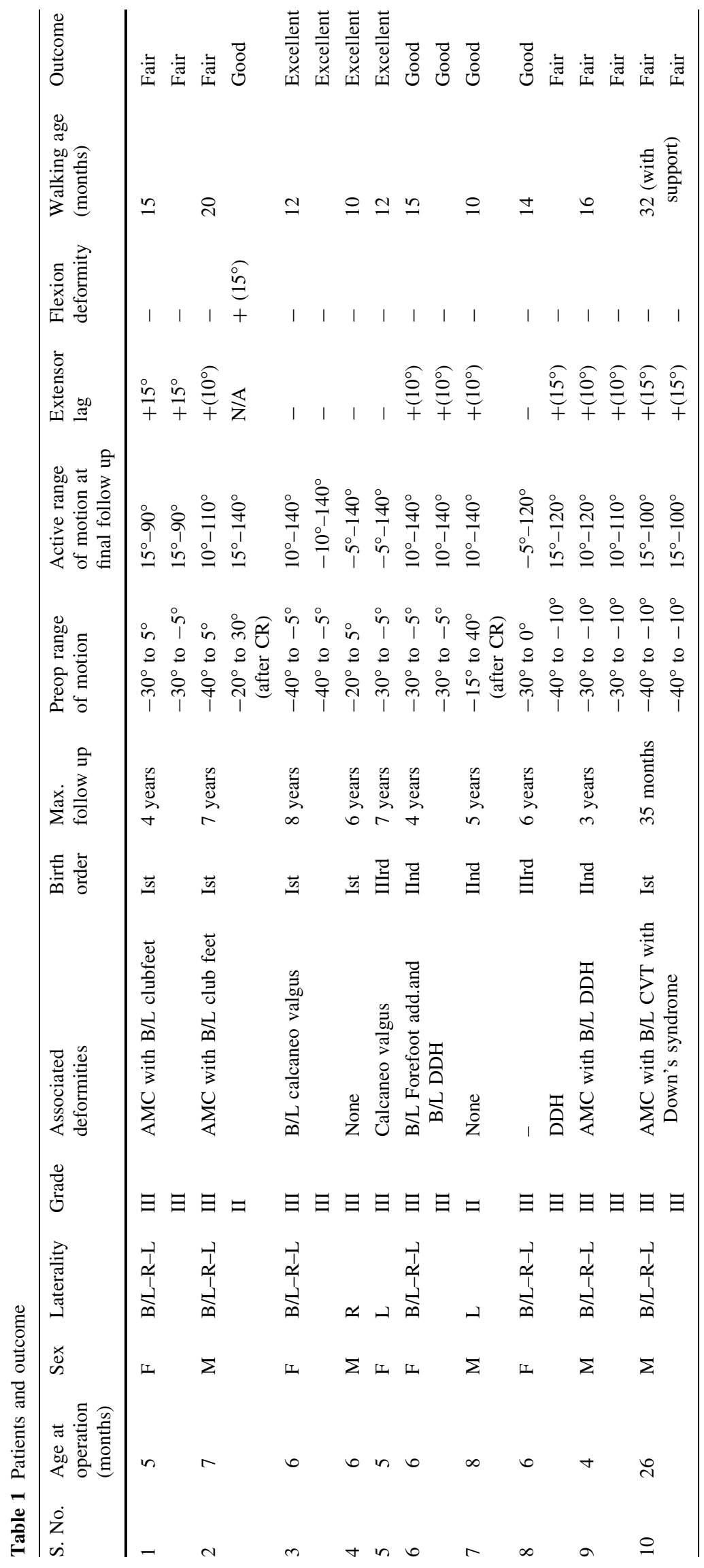


outcome is suggested; early treatment and the absence of any congenital anomalies may lead to a better outcome [8].

Three patterns of clinical presentation have been identified-isolated dislocation, multiple dislocations and syndromes [11]. The latter two are found to be most resistant to the conservative management as evident from the present series. Concomitant conditions and syndromes not only necessitate surgery but require multiple procedures and prolonged immobilisation in casts and braces, thereby lowering the functional status of the limb.

V-lengthening of the quadriceps tendon with anterior capsulotomy was used in all cases. This resulted in a stable reduction without need for any additional procedures [3]. The anterior cruciate ligament was found to be intact in all knees although it was long and atrophic [12]. There were no meniscal abnormalities except they were noted to be smaller in size, and the shape of femoral and tibial condyles was found normal. The patellae were small, indiscernible but with no evidence of concomitant dislocation. In our review of the literature, there were no references to additional intra-articular procedures performed to stabilise the knee joint.

Good function of the knee depends not only on stability and a good range of painless motion but also on the absence of residual deformities or muscle weakness which may lead suboptimal function. The functional classification given in the literature was thus modified to include flexion deformity, extensor weakness and the need for walking aids.

The incidence of flexion deformity and extensor weakness has not been elaborated in literature. Ferris and Aichroth reported a mild extensor lag of $10^{\circ}$ in one patient [1]. Ooishi et al. [9] described an extensor lag of $30^{\circ}$ in one out of three patients. Bell et al. reported an extensor lag of $30^{\circ}$ in four out of five of their patients [8].

The presence of a flexion deformity and extensor weakness correlated to multiple joint involvements in the present series (Table 1). Both the patients with clubfeet developed flexion deformities and extensor weakness. The cause may have been prolonged immobilisation in the flexed position secondary to recurrence and a relatively short gastrocnemius muscle that did not yield well to correction at both the ends. All patients with DDH and AMC also developed extensor weakness, probably due to short hamstrings [9]. In contrast, those patients with isolated knee involvement or postural deformities like calcaneovalgus foot (which corrected spontaneously) did not develop flexion deformities or extensor weakness. This may suggest the role of dynamic factors, e.g., the hamstring and gastrocnemius muscles producing extensor weakness by virtue of spanning two joints and having a strong flexor influence at the knee joint. Lengthening of the quadriceps tendon and immobilisation of the knee in a flexed position produces further weakening of an already abnormal muscle, thereby giving a power advantage to the hamstrings and gastrocnemius that may lead to an extensor lag or weakness. This advantage may not be clinically evident by tests done for over activity [13] because the hamstrings and gastrocnemius are essentially normal in such cases, neither having a contracture nor fibrosis of their substance. However, their strength increases substantially after lengthening of quadriceps tendon when an over-riding tibia is placed below the femoral condyles from reduction of the knee joint.

That extensor weakness has remained static over several years in our patients further supports the proposal that an essential cause of the lag is muscle imbalance that fails to improve due to fibrotic contracture of the quadriceps muscle. A fractional release of hamstrings [14] and gastrocnemius in patients undergoing V-lengthening of quadriceps (especially in AMC and multiple joint involvement in Grade III patients) may reduce the extensor lag by reducing effective strength of the respective muscles. Furthermore, it is pertinent to mention that those authors who have done only lengthening of hamstrings have not reported upon the occurrence or improvement of extensor weakness in their patients [1].

We conclude V-lengthening of the quadriceps tendon produces a satisfactory improvement in knee function for congenital dislocation of the knee. The theoretical role of the gastrocnemius and hamstring muscles in abetting flexion deformity and extensor weakness is suggested.

\section{References}

1. Ferris B, Aichroth P (1987) The treatment of congenital knee dislocation-a review of 19 knees. Clin Orthop 216:135-140

2. Jacobson K, Vopalecky F (1985) Congenital dislocation of the knee. Acta Orthop Scand 56:1-7

3. Curtis BH, Fisher RL (1969) Congenital hyperextension with anterior subluxation of the knee-surgical treatment and long term observations. J Bone Joint Surg Am 51-A:255-259

4. Katz MP, Grogono BJS, Soper KC (1967) The etiology and treatment of congenital dislocation of knee. J Bone Joint Surg Br 49B: $112-120$

5. Dobbs MB, Boehm S, Grange DK, Gurnett CA (2008) Case report: congenital knee dislocation in a patient with Larsen syndrome and a novel filamin B mutation. Clin Orthop Relat Res 466(6):1503-1509

6. Midellton DS (1935) The pathology of genu recurvatum. Br J Surg 22:696

7. Williams PF (1968) Quadriceps contracture. J Bone Joint Surg Br 50B:278-284

8. Bell MJ, Atkins RM, Sharrard WJW (1987) Irreducible congenital dislocation of the knee. Aetiology and management. J Bone Joint Surg Br 69B(3):403-406

9. Ooishi T, Sugioka Y, Matsumoto S, Fujii T (1993) Congenital dislocation of the knee-its pathological features and treatment. Clin Orthop 287:187-192

10. Laurence M (1967) Genu recurvatum congenitum. J Bone Joint Surg Br 49B:121 
11. Carlson DH, O'Connor J (1976) Congenital dislocation of the knee. Am J Roentgenol 127:465

12. Mayer L (1913) Congenital anterior subluxation of the knee: description of a new specimen, summary of the pathology of the deformity and discussion of its treatment. Am J Orthop Surg 10:411
13. Canale T (2003) Campbell's operative orthopaedics, 10th edn. Mosby, Philadelphia

14. Tachdjian MO (1990) Pediatric orthopaedics, 2nd edn. WB Saunders, Philadelphia 